\section{Unexpected functions}

It is assumed that most transcriptional activators function in the nucleus. In Cell, Roeder and colleagues show that OCA-B, a $B$ cell-specific coactivator first identified by its interaction with octamer-binding transcription factors, also regulates pre-B cell receptor signaling in the cytoplasm. OCA-B binds to and regulates the stability of Syk, a tyrosine kinase that transduces antigen receptor signals. OCA-B-deficient pre-B cells are hyper-responsive to interleukin 7 (IL-7) because of defective pre-B cell receptor signaling that triggers downregulation of the IL-7 receptor and promotes further B cell differentiation. OCA-B-deficient cells also contain less Syk; notably, this regulation occurs posttranslationally. OCA-B seems to block Syk phosphorylation, leading to increased proteasome-mediated degradation. OCA-B is thus a multifunctional regulator of $B$ cell development. $L A D$ Cell 125, 761-774 (2006)

\section{Anti-inflammatory pathway}

Carbon monoxide (CO) dampens inflammation in vitro and in vivo. In Immunity, Otterbein and colleagues investigate whether CO exerts immunosuppressive effects through a direct or an indirect mechanism. Microarray analyses show that $\mathrm{CO}$ inhibits lipopolysaccharide-induced expression of the Egr-1 transcription factor, whereas treatment with CO increases expression of the PPAR- $\gamma$ hormone receptor, an inhibitor of ischemia-induced Egr-1 expression, in the nuclei of macrophages. This increase in PPAR- $\gamma$ activity depends on the CO-induced generation of reactive oxygen species (ROS). Notably, PPAR- $\gamma$ activity is essential for the CO-mediated suppression of lipopolysaccharide-induced proinflammatory gene expression in macrophages in vitro and lipopolysaccharide-induced lipid peroxidation and leukocyte accumulation in lungs in vivo. Precisely how ROS promote PPAR- $\gamma$ activity remains to be determined, and whether PPAR- $\gamma$-independent mechanisms also contribute to the anti-inflammatory activity of $\mathrm{CO}$ is not known. However, these results align CO, ROS and PPAR- $\gamma$ in a pathway potentially capable of regulating immune homeostasis.

Immunity 24, 601-610 (2006)

\section{SOCS3, IL-23 and $\mathrm{T}_{\mathrm{H}^{-17}}$}

$\mathrm{T}$ helper cells that produce IL-17 ( $\mathrm{T}_{\mathrm{H}^{-17}}$ cells) are key regulators of inflammation. In the Proceedings of the National Academy of Science, Chen et al. show that suppressor of cytokine signaling 3 (SOCS3) inhibits the production of $\mathrm{T}_{\mathrm{H}^{-}}-17$ cells by suppressing signal transducer and activator of transcription 3 (STAT3). Using a conditional 'knockout' of SOCS3, they find that even conditions that normally drive specific $\mathrm{T}$ helper cell differentiation to non-IL17-producing $\mathrm{T}$ helper type 1 and 2 cells will, in the absence of SOCS3, drive $\mathrm{T}_{\mathrm{H}^{-17}}$ cell differentiation. Alternatively, conditions known to specifically enhance $T_{H^{-}}-17$ cell development, such as treatment with IL-23, IL- 6 and TGF- $\beta$, cause increased activation and binding of STAT3 to the promoters of two IL-17 genes, I/17a and $/ / 17 f$. These results demonstrate direct regulation of IL-17 production by STAT3, particularly after treatment with IL-23, and negative regulation by SOCS3.

Proc. Natl. Acad. Sci. USA 103, 8137-8142 (2006)

Research notes written by Christine Borowski, Douglas C. Braaten and Laurie A. Dempsey

\section{Innate autoimmune modifier}

Mice lacking the Fc $\gamma$ RIIB inhibitory receptor manifest autoimmune disease characterized by the production of chromatin-specific autoantibodies. The Y-linked autoimmune accelerator (Yaa) genetic modifier exacerbates this autoimmune phenotype and results in the production of antibodies specific for nucleolar rather than chromatin antigens. In Science, Bolland and colleagues show that the Btk tyrosine kinase is essential for Yaa-mediated autoimmune aggravation. Using a combination of microarray analysis and fluorescence in situ hybridization, they identify the genetic defect underlying the 'pro-autoimmune' effects of Yaa. The Y chromosome of $\mathrm{Yaa}^{+}$ mice contains a duplication of at least $17 \mathrm{X}$ chromosome genes, one of which encodes Toll-like receptor 7 (TLR7), which binds Btk. Treatment with a TLR7 agonist results in the production of nucleolar-specific autoantibodies in $\mathrm{Yaa}^{-} \mathrm{Fcgr}_{2 b^{-/-}}$mice, suggesting that excess TLR7 signaling is sufficient to recapitulate the $\mathrm{Yaa}^{+}$autoimmune phenotype and that seemingly minor increases in TLR7 expression may regulate autoimmune susceptibility. Science (18 May 2006) doi: 10.1126/science.1124978

\section{LAT microRNA}

The latency-associated transcript ( $L A T)$ of herpes simplex virus 1 (HSV-1) produces no known protein, yet its expression is associated with maintenance of latent infection of neurons by protecting them from apoptosis. In Nature, Gupta et al. demonstrate that $L A T$ encodes a microRNA that is necessary and sufficient for reducing apoptosis of infected cells by regulating their expression of transforming growth factor- $\beta$ (TGF- $\beta$ ) and the transcription factor SMAD3. Functional and computational studies determine that the first exon of $L A T$ encodes a microRNA and that deletion of the microRNA-LAT sequence from HSV-1 confers susceptibility to apoptosis of infected cells. TGF- $\beta$ and SMAD3, known regulators of cell growth and apoptosis, are shown to be targets of microRNALAT. Expression of TGF- $\beta$ - and SMAD3-specific siRNAs further demonstrates that 'knockdown' of their expression is sufficient for protecting cells from apoptosis. Thus, LAT regulates TGF- $\beta$ apoptosis signaling and thereby helps to maintain HSV-1 persistence.

Nature (31 May 2006) doi:10.1038/nature04836 $D C B$

\section{The MOZ squad}

Myeloid leukemia has been linked to translocations involving the gene encoding MOZ, a histone acetyltransferase. In Genes \& Development, Katsumoto et al. show that MOZ deficiency leads to embryonic lethality due to a severe defect in hematopoiesis. Although MOZ has been shown before to interact with the transcription factor AML1 (also called Runx1), the phenotype of MOZdeficient embryos more closely resembles that of embryos deficient in the transcription factor PU.1. MOZ-deficient embryos have fewer hematopoietic stem cells, no B cells and arrested erythrocyte development, but have an overabundance of myeloid lineage cells. MOZ also can physically interact with PU.1. Thus, by its ability to interact with both AML1 and PU.1, MOZ can profoundly influence blood cell formation.

$L A D$

Genes Dev. 20, 1321-1330 (2006) 